\title{
Empleo con apoyo para personas con enfermedad mental
}

\section{Supported empleoyment for persons with mental illness}

\section{FRANCISCO DE BORJA JORDÁN DE URRIES VEGA*}

\begin{abstract}
RESUMEN
El empleo con apoyo es una metodología de inserción laboral para personas con discapacidad con amplia evidencia basada en resultados. En el colectivo de personas con enfermedad mental su aplicación es reciente sobre todo en España. El artículo realiza una revisión en este campo extrayendo evidencias de la efectividad y varias implicaciones para la práctica del modelo en el campo de la enfermedad mental en varios niveles.
\end{abstract}

\section{PALABRAS CLAVE}

Empleo con apoyo, Enfermedad mental, Rehabilitación laboral.

\begin{abstract}
Supported employment is a job integration methodology for persons with disabilities with wide outcome based in evidence. For persons with mental illness the application has been more recently developed, especially in Spain. The article provides a revision of this field extracting evidences of effectiveness and practice implications in the area of mental illness at different levels.
\end{abstract}

\section{KEY WORDS}

Supported employment, Mental illness, Vocational rehabilitation.

\footnotetext{
*Instituto Universitario de Integración en la Comunidad (INICO), Universidad de Salamanca. bjordan@usal.es
} 


\section{INTRODUCCIÓN}

El empleo con apoyo está siendo empleado cada vez más con personas con enfermedad mental. Si bien la metodología del empleo con apoyo es clara y estructurada, y viene avalada por amplia literatura e investigación, hemos de ser conscientes de que en este colectivo se suman al conjunto de barreras sociales y laborales las creadas por el estigma de la enfermedad mental. A esto cabe añadir que la propia naturaleza de las diferentes patologías hace necesaria la adecuación de los puestos a la problemática de cada trabajador al objeto de que el trabajo sea una herramienta normalizadora y no un problema y que además, para cada persona, debemos saber gestionar adecuadamente los posibles momentos 0 periodos de crisis que puedan sobrevenir en función de las diversas patologias.

En lo laboral, y de manera concreta, los problemas peculiares que encontramos en las personas con enfermedad mental (Bellver, 2002) se centran en las dificultades en el aprendizaje y la realización del trabajo (como la dificultad en el procesamiento de la información, los problemas para iniciar una acción, o las dificultades de concentración, organización y planificación), la dificultades en las relaciones interpersonales (como el aislamiento social, la falta de confianza en sí mismos, la suspicacia/paranoia, y la variabilidad en los cambios de humor), y las dificultades en el ámbito físico (el estado de agitación, los trastornos del sueño, las dificultades de coordinación motriz, y la medicación que se esté tomando).

Ante esta situación, las prácticas tradicionales en rehabilitación laboral en el campo de la salud mental no han sido suficientemente efectivas para favorecer el empleo integrado (Bond y McDonel 1991; Bond et al. 2001; Cook y Razzano, 2000; Crowther et al., 2001), siendo habitual la falta de definición y estructuración de los programas sobre empleo existentes, que más que preparar a los usuarios de los servicios para el empleo competitivo, han servido para adaptar a los clientes a las alternativas de empleo que tienen las organizaciones. El empleo con apoyo es la única alternativa que puede aportar datos basados en la evidencia que apoyen su efectividad.

\section{LA EFICACIA DEL EMPLEO CON APOYO}

El empleo con apoyo ha demostrado reiteradamente su eficacia para poder afrontar el problema del acceso al empleo de las personas con enfermedad mental y mejorar situación. Bond et al., recogen en 2001 varios estudios que nos muestran, que gracias a la implementación del empleo con apoyo se produce un incremento en las tasas de empleo, no se encontraron resultados negativos (recaídas), los consumidores, personal y familiares apreciaron positivamente el cambio, los antiguos clientes de tratamiento de dia participaban más en la comunidad, y además los costes fueron menores. Otros autores como Fabian, (1992) reportan que la aplicación del empleo con apoyo produce mejoras significativas en la calidad de vida de personas con enfermedad mental. Igualmente, Floyd (2006), indican mejoras en satisfacción y autoestima, aumento de las relaciones sociales y participación en un rango amplio de puestos de trabajo.

Sin embargo, estos resultados no se obtienen porque el empleo con apoyo sea una panacea infalible para solucionar el problema, sino que es necesario tener en cuenta aspectos estructurales. La eficacia viene determinada por factores concretos (Becker et al., 2006) como los recursos y el personal de apoyo disponible, ya que cuantas más personas traba- 
jen en el servicio de empleo con apoyo más capacidad del servicio y más resultados. Por otro lado, es más importante ejecutar los componentes críticos de la práctica del modelo que adaptar el modelo a las condiciones locales. En este sentido, la situación del mercado local de trabajo y la fidelidad al modelo de empleo con apoyo tienen igual valor predictivo respecto a la posibilidad de acceder a un empleo.

Frecuentemente tendemos a achacar los resultados poco favorables a múltiples variables. Sin embargo, la investigación nos muestra (Perkins et al., 2005) cómo la mayoría de estos factores (género, edad, diagnóstico, historia de hospitalizaciones, meses desde el último empleo, espacio de tiempo mayor en que se ha mantenido un empleo, cuantía del Supplemental Security Income recibido) no parecen estar relacionados con que el individuo trabaje o no. Así mismo, el tiempo que el proveedor de servicios de empleo con apoyo dedica a viajar, entrenar, y asesorar a los desempleados sí parece estar relacionado de manera independiente con la posibilidad de obtener un empleo competitivo.

Por otro lado, también sabemos que los usuarios de los servicios encuentran y mantienen empleo con más facilidad (Schneider et al., 2002) cuando han trabajado anteriormente, tienen actitudes positivas hacia el trabajo, se les coloca lo antes posible en un trabajo de su elección, reciben preparación dirigida al trabajo en vez de entrenamiento general, reciben apoyo continuado en su trabajo, participan de manera activa en la intervención para el empleo, y no tienen pérdidas económicas como resultado de trabajar.

Pero no sólo los factores anteriores aumentan la efectividad de empleo con apoyo. La experiencia demuestra
(Schneider et al., 2002) que los servicios de empleo parecen ser más efectivos cuando funcionan de manera integrada con los equipos de salud mental. La integración de los servicios de empleo y los de salud mental (Drake et al., 2003) consigue que los programas tengan más éxito en mantener a sus clientes en servicios de empleo (un 50\% abandonaban el programa al inicio o las pocas semanas frente a un $90 \%$ que continuaban varios meses o hasta obtener un empleo integrado). Además, se han podido identificar causas del abandono de los programas tales como las pobres habilidades sociales, los sentimientos de recha$z 0$, los efectos colaterales de la medicación, las dificultades familiares, los problemas de transporte, el desarrollo de procesos excesivamente largos por los servicios, la duplicidad de evaluaciones que ya se les han realizado, y el no prestar atención a las preferencias del usuario.

Los servicio de empleo integrados con los de salud mental no solamente tienen efectos positivos en los usuarios de los servicios, sino también en los profesionales de los mismos (Drake et al., 2003), generando cambios en las actitudes, ideas, y comportamientos de los clinicos escépticos provenientes del campo de la salud mental y consiguiendo una evolución positiva en su sensibilización hacia el empleo al observar que los usuarios encuentran satisfacción en el empleo, optimismo, aumento de la autoestima y otras mejoras. Así, empiezan a recomendar a los usuarios el empleo como opción que les puede proporcionar beneficios y a participar activamente en ayudar a los usuarios a elegir, encontrar y mantener un empleo. Además, las planificaciones y estrategias se completan con más y mejor información clínica y se añade mucha más información relevante para la planificación del empleo, la búsqueda y emparejamiento, y el apoyo en 
el puesto. Sin embargo, no son estos profesionales los únicos beneficiados, también los especialistas en empleo evolucionan positivamente ya que desarrollan conocimiento clínico y habilidades, aprenden sobre la enfermedad mental, sobre sus tratamientos, las estrategias de afrontamiento y los posibles sistemas de apoyo.

\section{LA SITUACIÓN EN ESPAÑA}

En España y Europa empiezan también a desarrollarse iniciativas de empleo con apoyo que consiguen introducir personas con enfermedad mental en el mercado laboral ordinario (Jordán de Urries, Verdugo y Vicent, 2005; Jordán de Urríes, Beyer y Verdugo, 2008; Verdugo y Jordán de Urries, 2003) que aparecen con cierta timidez como la desarrollada desde la Comunidad de Madrid aplicando el modelo tradicional de empleo con apoyo a un grupo de personas con enfermedad mental (Esteban y Jordán de Urries, 2006). La comparación de esta iniciativa con un programa similar aplicado a un grupo de personas con discapacidad intelectual, objetivo más tradicional del empleo con apoyo, mostró grandes similitudes, y algunas diferencias, ya que en el colectivo de personas con enfermedad mental el apoyo se realizaba con mayor énfasis fuera del lugar de trabajo. Además, la tendencia general era ocultar la discapacidad del trabajador en la empresa para evitar la estigmatización, mientras que en el colectivo de personas con discapacidad intelectual esto no ocurría.

El programa ECA Caja Madrid, promovido por la Obra Social de Caja Madrid y gestionado por el Instituto Universitario de Integración en la Comunidad, INICO, ha supuesto también un considerable impulso para el empleo con apoyo en España (Jordán de Urries y
Verdugo, 2007a). La Confederación Española de Agrupaciones de Familiares y Enfermos Mentales, FEAFES, accedió al programa en el año 2006 participando con casi 60 acciones de empleo con apoyo entre 2006 y 2007.

Al impulso aportado por ECA Caja Madrid es de esperar que se sume el que aportará la reciente regulación del empleo con apoyo en España (Jordán de Urries y Verdugo, 2007b) mediante el Real Decreto 870/2007 de 2 de julio de 2007, aunque hasta el momento no parece que el Decreto se esté desarrollando como sería deseable, manteniéndose al respecto por el momento un alto grado de incertidumbre.

\section{ALGUNAS IMPLICACIONES}

La experiencia acumulada, las evidencias y los resultados, deben servir para generar cambios en las organizaciones, los gestores, y los profesionales que desarrollan los servicios. Para conseguir los efectos deseados de cara al mayor beneficio de los usuarios (Bond et al., 2001), las organizaciones deben establecer un claro compromiso con el empleo competitivo como meta posible para sus clientes con enfermedad mental. Es necesario que adopten un enfoque rápido de búsqueda de empleos, como el desarrollado por el empleo con apoyo, para ayudar a obtener directamente el trabajo a sus clientes (prescindir de entrenamientos previos y de largas evaluaciones). Las ubicaciones laborales deberán ser individualizadas según las preferencias, capacidades y experiencia laboral de los clientes, y el acompañamiento y los apoyos deben mantenerse indefinidamente con la intensidad necesaria en cada momento. Por último, el equipo de profesionales de empleo con apoyo deberá estar muy integrado con el equipo de tratamiento de salud mental. 
En lo que respecta a los gestores (Schneider et al., 2002), deberán utilizar el empleo como indicador clave de ejecución del conjunto de sus servicios. Para ello deben asegurar el acceso a un amplio rango de empleos para las personas con problemas de salud mental y especificar la inclusión social como criterio en los resultados de empleo. Además, se procurará la intervención temprana en los diferentes problemas de salud mental, dirigida a la permanencia en el empleo para quienes lo tengan en el momento de detectarse una patología. Para ello será necesario integrar los equipos de empleo con apoyo con los equipos de salud mental y transformar los centros de día para proporcionar empleo con apoyo, entrenando a los profesionales en los principios del empleo con apoyo como una intervención basada en evidencias. La utilización de herramientas como la "Supported Employment Fidelity Scale" (Bond et al., 1997; Bond et al. 2001) o los Estándares de Calidad de la Unión Europea de Empleo con Apoyo (Evans et al., 2005), y el instrumento que los operativiza (Jordán de Urries y Verdugo, 2006), serán una base adecuada para el desarrollo de servicios de empleo con apoyo. Y todas las propuestas anteriores serán tanto más efectivas en tanto se consiga trabajar de manera colaborativa y sinérgica con las diferentes organizaciones y la administración.

En tercer lugar y en lo que respecta a las implicaciones para los profesionales (Schneider et al., 2002), deberán evaluar las habilidades laborales de los usuarios en su admisión al tratamiento. De esta manera podrán dirigir rápidamente a los usuarios a un especialista en empleo con apoyo el cual a su vez debe tener acceso a consultas con expertos en salud mental. Conjuntamente deberán asegurar métodos efectivos para tratar sintomas negativos, previniendo la pérdida de habilidades sociales de los usuarios, pro- moviendo su adquisición y construyendo la confianza y habilidades de los usuarios en relación al empleo como parte de su tratamiento rehabilitador.

A las consideraciones anteriores debemos sumar otras implicaciones referidas a la práctica profesional. Esta habrá de basarse en una adecuada planificación centrada en la persona para la que será también necesaria una evaluación en ambientes reales desde una perspectiva ecológica y con el objetivo del empleo, que nos sirva para desarrollar un perfil del cliente, minimizando limitaciones y maximizando capacidades. Además, deberá aplicarse un grado adecuado de flexibilidad en el diseño y búsqueda de puestos de trabajo, adaptando las fórmulas de contrato, horarios y salarios por rendimiento, de manera que el trabajo no sea un problema para el trabajador sino un beneficio. Para evitar el aumento del estigma y el rechazo de los usuarios de la figura del profesional de apoyo, se deberán utilizar los apoyos naturales como herramientas de integración en el puesto, minimizando la presencia del especialista (los compañeros de trabajo serán una pieza clave en la detección de indicios de crisis o en la gestión de las mismas). Para todo ello, el profesional del empleo que desarrolle su actividad con personas con enfermedad mental habrá de tener un sólido conocimiento de la enfermedad mental y de las diferentes patologías, así como de la persona y de su patología concreta. Deberá disponer igualmente de capacidad para apoyar en diferentes áreas, de formar al trabajador, compañeros y supervisores, y de realizar un marketing efectivo y una "venta positiva" de las capacidades y solvencia de los trabajadores a los que representa.

Además, la formación profesional de los candidatos es en ocasiones cualificada, y los posibles puestos a los que pue- 
den optar demandan de preparadores con igual cualificación (Esteban y Jordán de Urries, 2006) lo cual es difícil de encontrar para cada puesto, siendo pues crucial implicar directamente a profesionales de la empresa para la formación y apoyo en el puesto, a quienes se deberá preparar para poder detectar sintomas premórbidos, indicadores de una posible recaída de su compañero, que puedan detectar indicios de crisis para minimizar su repercusión en el empleo.

Respecto a la familia, su papel es fundamental en el apoyo hacia el empleo integrado aunque no siempre es un agente de apoyo (Esteban y Jordán de Urries, 2006). La metodología de ECA para personas con enfermedad mental debe informarles adecuadamente sobre el papel del trabajo en la vida de sus familiares; dificultades para la integración laboral de personas con problemas psiquiátricos crónicos; papel de la rehabilitación laboral; actualidad del mercado laboral; riesgos de la incorporación laboral como agravamiento de la sintomatología, recaidas, abandono de la medicación, perdida y recuperación de pensiones, etc. Para ello debe conseguirse la participación de la familia en el proceso de inserción laboral y en reuniones informativas donde se les proporcione toda esta información. Las familias suelen desconocer estos datos y, por tanto, oponerse al acceso al empleo de su familiar ante el temor a la pérdida de la pensión de por vida o de la gratuidad de plaza en determinados centros.

Para acabar, y en lo que concierne a la administración, el desarrollo efectivo del Real Decreto 870/2007 es un elemento crucial para el desarrollo del empleo con apoyo en nuestro país. Una vez disponemos de la norma, con todo lo que supone de positivo y algunas carencias (Jordán de Urríes y Verdugo, 2007b) es crucial hacerla efectiva y operativizarla para que las organizaciones promotoras de servicios puedan aprovecharse de la misma cuanto antes. 


\section{BIBLIOGRAFÍA}

Becker, D. R., X. H., McHugo, G. J., Halliday, J., y Martinez, R. A. (2006). What predicts supported employment program outcomes? Community Mental Health Journal, 42 (3), 303-313.

Bellver, F. (2002). Peculiaridades del empleo con apoyo aplicado a las personas con enfermedad mental. Interpsiquis 2002, 3er Congreso Virtual de Psiquiatría, Actas del congreso. (On line).

Bond, G. R., Becker, D. R., Drake, R. E., Rapp, C. A., Meisler, N., Lehman, A. F., Bell, M. D., y Blyler, C. R. (2001). Implementing supported employment as an evidenced based practice. Psychiatric Services, 52 (3), 313-322.

Bond, G. R., Becker, D. R., Drake, R. E., y Vogler, K. M. (1997). A Fidelity Scale for the Individual Placement and Support Model of Supported Employment. Rehabilitation Counseling Bulletin, 40 (4), 265-284.

Bond, G. R., y McDonel, E. C. (1991). Vocational rehabilitation outcomes for persons with psychiatric disabilities: An update. Journal of Vocational Rehabilitation, 1 ( 3), 9-20.

Cook, J., y Razzano, L. (2000). Vocational rehabilitation for persons with schizophrenia: Recent research and implications for practice. Schizophrenia Bulletin, 26, 87-103.

Crowther, R. E., Marshall, M., Bond, G. R., y Huxley, P. (2001). Helping people with severe mental illness to obtain work: Systematic review. British Medical Journal, 322, 204-208.

Drake, R. E., Becker, D. R., Bond, G. R., y Mueser, K. T. (2003). A process analisys of integrated and non-integradted approaches to supported employment. Journal of Vocational Rehabilitation, $18,51-58$.

Esteban, B., y Jordán de Urríes, F. B. (2006). Empleo con apoyo para personas con discapacidad intelectual y para personas con enfermedad mental. Comparación metodológica en dos proyectos piloto. Siglo Cero, 37 (2), 63-78.

Evans, M., Lopes, I., Katsouda, C., Diepeveen, A., Fleming, M., Haddock, M., Hazzard, T., Schuller, S., Johnova, M., Boxava, P., Martínez, S., Jordán de Urries, F. B., Eklund, B., Sariola, L., y Haltunen, H. (2005). Information booklet and quality standards. Belfast: European Union of Supported Employment.
Fabian, E. (1992). Supported employment and the quality of life: does a job make a difference? Rehabilitation Counseling Bulletin, 36 (2), 84-97.

Floyd, M., Grove, B., Schneider, J., Secker, J., Boyce, M., Jhonson, R., y Slade, A. (2006). Social inclusion through employment support for adults with mental illnes.

Jordán de Urries, F. B. Beyer, S. y Verdugo, M. A. (2008). Estudio comparativo del empleo con apoyo en Europa. En 2n. Premi Investigació $i$ innovació sobre persones amb Discapacitat Intel-Lectual. Treballs premiats 2008. Pp. 116-194. Manresa: AMPANS.

Jordán de Urries, F. B., Verdugo M. A. y Vicent, C. (2005). Análisis de la evolución del empleo con apoyo en España. Madrid: Real Patronato sobre Discapacidad.

Jordán de Urries, F. B., y Verdugo, M. A. (2006). Principios éticos y estándares de calidad para inserción en empleo ordinario mediante Empleo con Apoyo. El modelo de la EUSE. Intervención Psicosocial, 14 (3), 343-354.

Jordán de Urríes, F. B., y Verdugo, M. A. (2007a). Aportaciones significativas recientes en empleo con apoyo desarrolladas desde el INICO. M. A. Martínez, F. B. Jordán de Urríes, C. Cerda, y J. Gozalbez (Coordinadores), El camino hacia la inclusión social. VIII Simposio Internacional de Empleo con Apoyo (pp. 107-113).

Jordán de Urríes, F. B., y Verdugo, M. A. (2007b). Empleo con Apoyo: el nacimiento de una regulación muy esperada. Alares, (2), 2-8.

Perkins, D. V., Born, D. L., Raines, J. A., y Galka, S. W. (2005). Program evaluation from an ecological perspective: Supported employment services for persons with serious psychiatric disabilities. Psychiatric Rehabilitation Journal, 28 (3), 217-224.

Schneider, J. , Heyman, A., y Turton, N. (2002). Employment for people with mental health problems. Expert Briefing, (Summer 2006), 1-6.

Verdugo, M. A., y Jordán de Urries, F. B. (2003). Empleo con apoyo y salud mental. M. A. Verdugo, D. López, A. Gómez, y M. Rodríguez (Coordinadores), Atención comunitaria, rehabilitación y empleo (pp. 153-164). Salamanca: Instituto Universitario de Integración en la Comunidad. 